\title{
An 8-year study on mycoplasma in rheumatoid arthritis
}

\author{
ELLI JANSSON, P. MÄKISARA, K. VAINIO, U. VAINIO, O. SNELLMAN, \\ AND SIRKKA TUURI
}

The Rheumatism Foundation Hospital, Heinola, Aurora Hospital, and the Invalid Foundation Hospital, Helsinki, Finland

In the winter of 1962-1963 we studied Mycoplasma pneumoniae infections in Helsinki, and our attention was drawn to the autoimmune phenomena, e.g. cold agglutinins, occurring in this disease. It led us to investigate the possible role of mycoplasma in rheumatoid arthritis (RA) as a primary trigger initiating the rheumatoid process. A summary of this 8-year study is presented here.

\section{Material and methods}

PATIENT SERIES

I. In 1963-1964, 28 specimens of synovium or joint fluid were studied from patients with RA under treatment in the Rheumatism Foundation Hospital at Heinola.

II. In 1966-1967, 33 specimens of joint fluid from patients seen at a Rheumatism Outpatient Clinic in Helsinki were examined.

III. In 1967-1968, 27 specimens of synovial fluid and tissue from patients treated at Heinola and in Helsinki were studied.

As controls, 24 specimens of synovium from patients with traumatic joint lesions and under treatment in the Invalid Foundation Hospital were examined simultaneously with Series II and III.

ISOLATION TECHNIQUE

This is reported in detail elsewhere. During the first study, conventional mycoplasma media were used (Jansson and Wager, 1967). In the second study, inoculation into yolk sacs of embryonated hens' eggs was also performed (Jansson, Vainio, Snellman, and Tuuri, 1971). In the third study brain heart infusion broth was used instead of PPLO broth. The broth medium was further enriched with egg yolk pasteurized at $+60^{\circ} \mathrm{C}$. for $50 \mathrm{~min}$.; $0.1 \mathrm{ml}$. egg yolk was added to $10 \mathrm{ml}$. broth. The broth culture tubes were incubated for 20 days and three passages in broth were performed (Jansson, Mäkisara, Vainio, Snellman, and Tuuri, 1971). By this time modern microscopic equipment was available, a Leitz Orthoplan microscope with an Achr. 70/L4, which corrects the thickness of the Dienes-stained agar block preparations. The microbiological difficulties in these studies have already been discussed (Jansson, 1971).

The methods used for the biochemical tests, preparation of hyperimmune sera and immune ascites, growth inhibition test, and antibody studies by the indirect haemagglutination test have also been described $\hat{\omega}$ elsewhere (Jansson and Wager, 1967; Jansson, Vainio, $ᄋ$ and others, 1971; Jansson, Mäkisara, and others, 1971).

\section{Results}

\section{Isolation studies}

In the first study a mycoplasma was recovered in $\mathbb{D}$ two cases out of 28 , in the second in eleven out of 33 , $\frac{}{\mathbb{D}}$ and in the third in each out of 27 cases; 24 synovial 3 specimens of traumatic joint lesions studied simul- $\mathbb{\Phi}$ taneously by the same methods were negative for $\overrightarrow{0}$ mycoplasma.

As a rule, the colonies were tiny, about $1 / 10$ $1 / 100$ of the large-colony mycoplasma. On prima isolation on cell-free media, six strains showed the typical 'fried-egg' appearance, which four of them lost in subcultures. In later subcultures on enriched $\frac{0}{8}$ brain heart infusion agar with $1 \cdot 1$ per cent. agar, $\stackrel{\mathbb{Q}}{\circ}$ they tended to form an abundant fragile growth. $\overrightarrow{\overrightarrow{0}}$

The isolates did not convert into bacteria when 3 penicillin and thallium acetate were omitted. They required sterol for their growth. On primary? isolation all the strains grew better in an anaerobic milieu and rather slowly, 7 to 20 days. With high? magnification $(\times 1,000)$ and an oil immersion lens, 3 their fragile structure with small pleomorphic granular elements of the Dienes-stained colonies could be seen. Figs 1 to 4 (opposite) illustrate the microscopical appearance of some isolates.

The isolates did not ferment glucose nor did they $\frac{?}{0}$ split urea; they metabolized arginine, but this property was often lost when many subcultures $N$ had been done.

\section{Growth inhibition studies}

The first two isolates (14-P and 20-P) in 1964 were identified as $M$. arthritidis, which was confirmed by Lemcke (1969) and Hayflick (1970). Rabbit antisera prepared against eleven isolates and immune ascites against nineteen isolates were tested againstō 

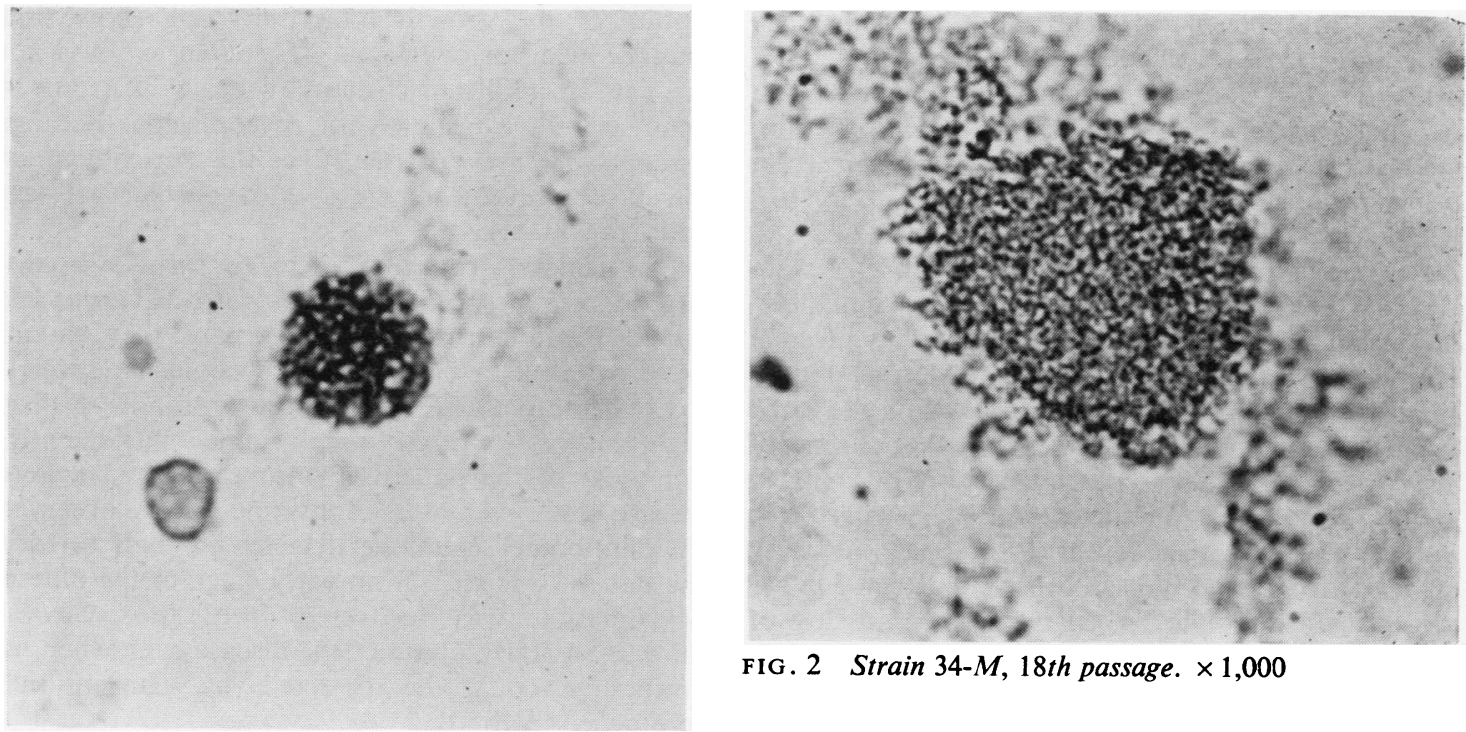

FIG. 1 Strain $189-M, 5$ th passage. $\times 540$

FIG. 2 Strain $34-M, 18$ th passage. $\times 1,000$

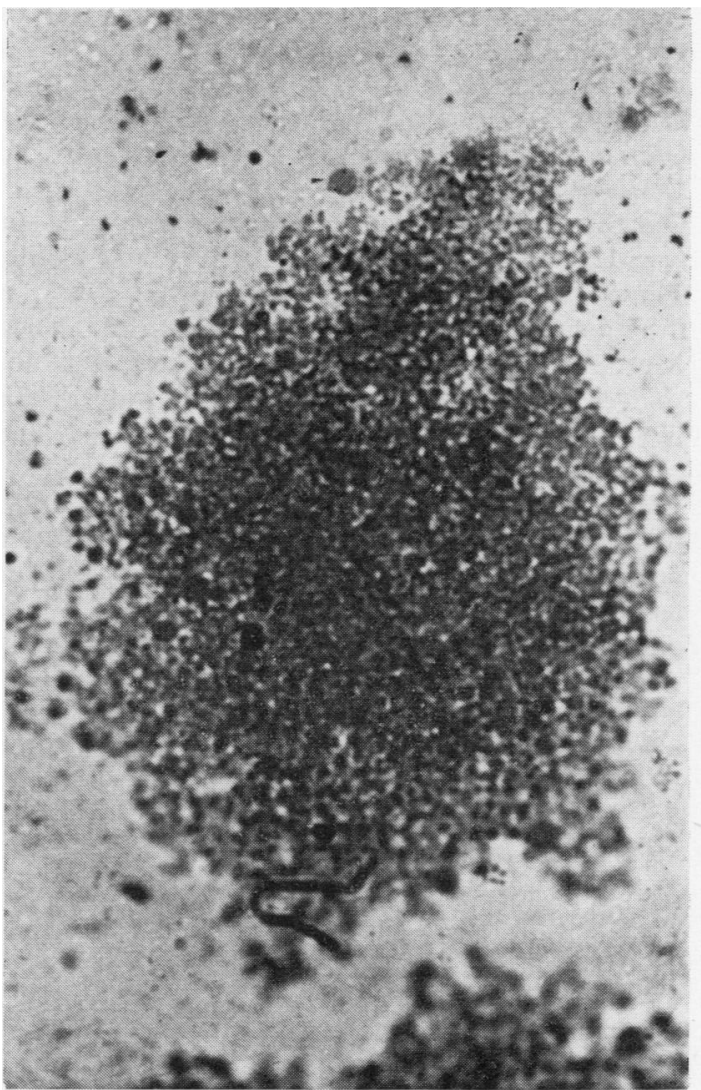

FIG. 4 Strain 138-M, 9th passage. $\times 1,000$
FIG. 3 Strain $146-M$, 9 th passage. $\times 1,000$

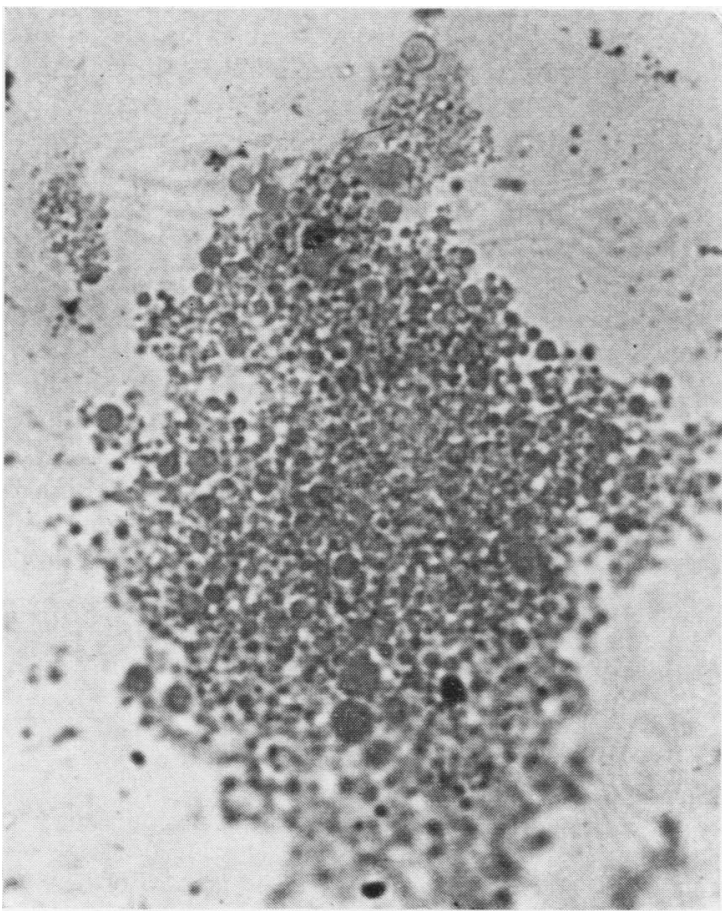

FIO. 1 to 4 Isolates from rheumatoid arthritis stained with Dienes stain.

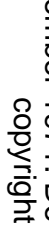

응

$+$ 
known mycoplasma species: $M$. hominis, $M$. salivarium, $M$. fermentans, $M$. orale type $1, M$. arthritidis, $M$. pulmonis, and $M$. gallinarum. There was a very narrow inhibition zone of 1 to $3 \mathrm{~mm}$. against $M$. arthritidis and no inhibition against the other mycoplasma mentioned.

\section{Antibody studies}

As a rule, no antibodies against isolate 20-P were detected by the complement-fixation or metabolic inhibition tests. A boy aged 18 years with juvenile RA showed an antibody titre of 25,000 measured by the indirect haemagglutination test. Using the same technique and another isolate, 176-M, 30 per cent. of 421 Waaler-Rose-positive sera (in a titre of $\geqslant 1: 128$ ) showed antibodies in titres $\geqslant 1: 8$, usually low.

In 1970, a girl aged 2 years was seen in an early stage of juvenile RA. Antibodies were detected in her serum against isolate 176-M from RA in a titre of 1:1,024 and against isolate 71-T in a titre of 1:512. Strain 71-T was recovered in our laboratory from the urethral specimen of a male with nongonococcal urethritis (NGU) (Jansson, Lassus, Stubb, and Tuuri, 1971). A man aged 40 years with acute arthritis and purpura had antibodies against this strain in a titre of $1: 512$ and against $M$. arthritidis in a titre of 1:64. A mycoplasma related to $M$. arthritidis was cultivated from his synovial fluid (Jansson, Vainio, Lassus, and Tuuri, to be published). Discussion

Our results suggest that the isolation of mycoplasma was dependent on the culture medium and method. These fastidious human mycoplasma grew very infrequently on conventional mycoplasma media. Moreover, their size is so small and their morphology so pleomorphic that they are probably overlooked by scientists who are used to studying the largecolony mycoplasma.

Eaton's success in isolating in embryonated hens eggs the agent of primary atypical pneumonia, now called $M$. pneumoniae, led us to try the same technique. In our opinion, the addition of fresh pasteurized egg yolk to mycoplasma broth medium was an important improvement. This could of course be criticised by asking whether it might introduce an avian mycoplasma into the medium. If it did so, it would probably be the glucosefermenting $M$. gallisepticum, colonies of which are much larger than those of our isolates. The results of the growth inhibition tests show that another avian mycoplasma, $M$. gallinarum, was not present. In addition, cultures of egg yolk from 24 hens eggs were negative for mycoplasma. Because mycoplasma are heat-sensitive, the enrichment of $\stackrel{\vec{\omega}}{\mathscr{}}$ the broth culture medium with pasteurized egg $\bar{c}$ yolk is probably safe.

Our isolates from RA seem to form a homo- $\frac{\bar{\omega}}{\frac{6}{6}}$ geneous group sharing antigens with $M$. arthritidis $\stackrel{\mathbb{D}}{\circ}$ and a T-strain mycoplasma. Perhaps they should be regarded as a new human mycoplasma species.

Concerning the results of the antibody studies, high antibody titres were found infrequently, but most of the patients in our series had a long disease history. In cases of juvenile RA we observed that antibodies could be detected in high titres if $\dot{\omega}$ the patient was seen at an early stage of the illness. Mycoplasma was recovered from the synovial or fluid in an active phase of the disease even when no 8 antibodies could be detected by conventional 은 serological methods.

This 8-year study indicates that mycoplasma may $\infty$ be implicated in the pathogenesis of RA. We hope that other scientists will try to confirm our results. Summary

When conventional cell-free culture media were used in 1963-1964, a mycoplasma was recovered in two out of 28 cases with rheumatoid arthritis A modified cultivation method was developed anf also a modern large view-field microscope later became available. By this technique a mycoplasma was isolated in every one of 27 patients studied. 24 synovial specimens from cases of traumatic joint lesions were investigated simultaneously by the same methods and were found to be negative for mycoplasma. The isolates from rheumatoid arthritis seem to form a homogeneous group sharing antigens with $M$. arthritidis and a T-strain mycoplasma. Antibodies against one isolate could be detected in high titres by the IHA technique if 0 the patient was seen at an early stage of the illness. Mycoplasma was recovered from the synovial fluid or tissue from patients in an active phase of $₹$ the disease even when no antibodies could be $\frac{9}{9}$ detected by the conventional serological methods. $D$ This study was supported by grants from the Sigrid Jusélius Foundation, the Finnish Medical Research $N$ Council and the University of Helsinki.

\section{References}

Hayflick, L. (1970) Personal communication.

Jansson, E. (1971) J. clin. Path., 24, 53 (Isolation of fastidious mycoplasma from human sources).

-, Lassus, A., Stubb, S., AND Tuuri, S. (1971) Brit. J. vener. Dis., 47, 122 (Studies on T-strain mycoplasma in nongonococcal urethritis).

—, Mäkisara, P., Vainio, K., Snellman, O., ANd TuURI, S. (1971) Acta rheum. scand., 17, in press (Further studies on mycoplasma in rheumatoid arthritis).

-

- - S - SNellman, O., AND TUURI, S. (1971) Ann. rheum. Dis., 30, in press (Search for mycoplasma in rheumatoid arthritis)

- AND WAGER, O. (1967) Ann. N.Y. Acad. Sci., 143, 535 (Mycoplasma in collagen diseases and blood dyscrasia) LeMCKE, R. (1969) Personal communication.

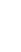

